\title{
Eigenvalue solution of thermoelastic instability problems using Fourier reduction $\dagger$
}

\author{
BY YUN-BO YI ${ }^{1}$, J. R. BARBER ${ }^{1}$ AND P. ZAGRODZKI ${ }^{2}$ \\ ${ }^{1}$ Department of Mechanical Engineering and Applied Mechanics, \\ University of Michigan, Ann Arbor, MI 48109-2125, USA \\ ${ }^{2}$ Raytech Composites Inc., 1204 Darlington Avenue, Crawfordsville, IN 47933, USA
}

Received 3 February 2000; accepted 5 June 2000

A finite-element method is developed for determining the critical sliding speed for thermoelastic instability of an axisymmetric clutch or brake. Linear perturbations on the constant-speed solution are sought that vary sinusoidally in the circumferential direction and grow exponentially in time. These factors cancel in the governing thermoelastic and heat-conduction equations, leading to a linear eigenvalue problem on the two-dimensional cross-sectional domain for the exponential growth rate for each Fourier wavenumber. The imaginary part of this growth rate corresponds to a migration of the perturbation in the circumferential direction.

The algorithm is tested against an analytical solution for a layer sliding between two half-planes and gives excellent agreement, for both the critical speed and the migration speed. Criteria are developed to determine the mesh refinement required to give an adequate discrete description of the thermal boundary layer adjacent to the sliding interface. The method is then used to determine the unstable mode and critical speed in geometries approximating current multi-disc clutch practice.

Keywords: thermoelastic instability; perturbation methods; multi-disc clutches; brakes

\section{Introduction}

When two bodies slide against each other, frictional heat is generated and the resulting thermoelastic deformation alters the contact pressure distribution. This coupled thermomechanical process is susceptible to thermoelastic instability (TEI). Above a certain critical speed, a nominally uniform pressure distribution is unstable, giving way to localization of load and heat generation and hence to hot spots at the sliding interface (Barber 1969; Kennedy \& Ling 1974; Floquet \& Dubourg 1994; Bryant et al. 1995; Kao et al. 2000). The problem is particularly prevalent in energy-dissipation systems such as brakes and clutches. Hot spots can cause material damage and wear and are also a source of undesirable frictional vibrations, known in the automotive disc brake community as 'hot roughness' or 'hot judder' (Kreitlow et al. 1985; Inoue 1986; Zagrodzki 1990; Anderson \& Knapp 1990; Lee \& Dinwiddie 1998).

$\dagger$ The authors dedicate this paper to the memory of Dr R. A. Burton, whose pioneering work made possible modern methods of analysis of thermoelastic instability. 


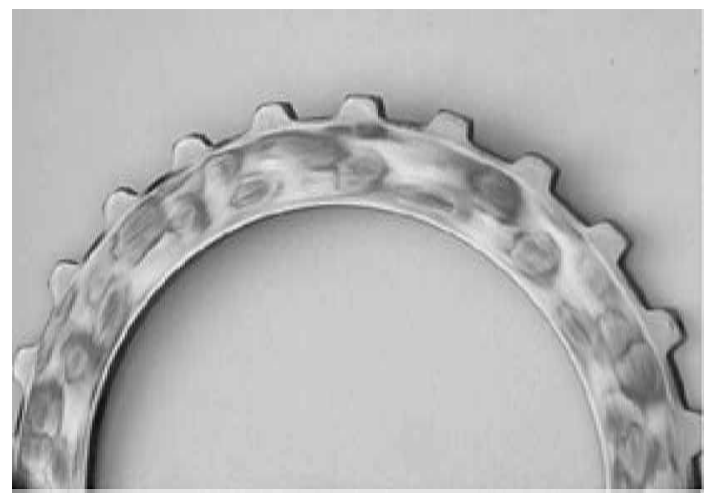

Figure 1. Evidence of hot spotting on a clutch disc.

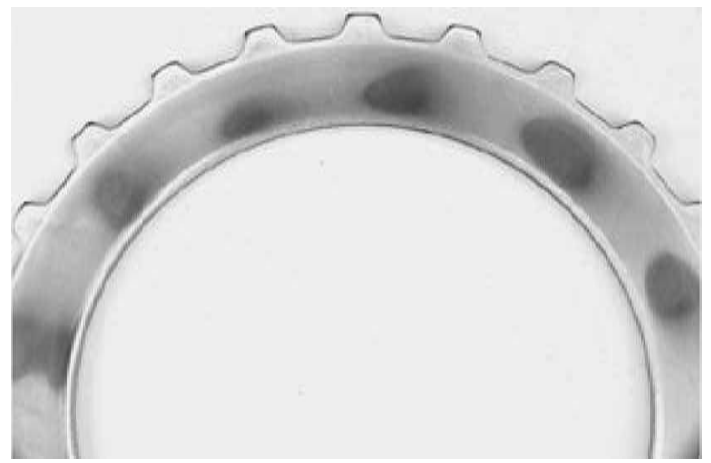

Figure 2. Clutch disc after a single engagement.

Figure 1 shows one of the plates of a typical multi-disc wet clutch after a period of normal service. The dark areas correspond to regions in which high local temperatures have been experienced. Evidence of surface melting can be found in extreme cases. In addition, transfer of friction material components and the products of overheated transmission fluid may be involved. The pattern seen in figure 1 represents the effect of multiple engagements of the clutch and shows several series of hot spots at different locations overlayed on each other. A better picture of the phenomenon is obtained if the clutch is examined after a single engagement, in which case the regular pattern of figure 2 is obtained. The complete disc in this particular case exhibits 12 equally spaced hot spots on each side and they are arranged antisymmetrically. In other words, the hot spots on the opposite side of the disc are located in the gaps between those shown in the figure.

\section{(a) Stability analysis}

Burton et al. (1973) used a perturbation method to investigate the stability of contact between two sliding half-planes. The system is linearized about the uniform pressure state and perturbations that can grow exponentially with time are sought. Their results provided useful insight into the nature of the phenomenon, but there is no inherent length-scale in the problem as defined and it was found that sufficiently long wavelengths are always unstable. A length-scale can be artificially introduced into the analysis by restricting attention to perturbations below a certain wavelength, 
estimated as being comparable with the linear dimensions of the practical system, but the resulting predictions for critical speed do not generally show good agreement with those observed experimentally (Dow \& Stockwell 1977; Banerjee \& Burton 1979).

\section{(b) The effect of geometry}

The first solution of a TEI problem involving a geometric length-scale was given by Lee \& Barber (1993), who used Burton et al.'s (1973) method to analyse the stability of a layer sliding between two half-planes. This geometry provided a first step towards that of the typical disc brake assembly, where a disc slides between two pads of a friction material. Using typical material properties from automotive applications, it was found that stability is governed by a deformation mode that is antisymmetric with respect to the mid-plane of the layer and that has a wavelength proportional to the layer thickness.

Despite the considerable idealizations involved in Lee's theory, it provides plausible predictions for the critical speed and the mode shape in typical brake assemblies and is therefore quite widely used in the brake and clutch industry for TEI analysis. However, there is a clear need for a method that will account for other features of the system geometry, such as the finite width of the sliding surface, the axisymmetric geometry of the disc, and the 'hat' section used to attach the disc to its support. One approach is to use finite-element analysis (FEA) to solve the coupled transient thermoelastic contact problem in time (Zagrodzki 1990; Johansson 1993; Zagrodzki et al. 1999). This method is extremely flexible, in that it can accommodate nonlinear or temperature-dependent constitutive behaviour, more realistic friction laws, and practical loading cycles. However, it is also extremely computer intensive and appears unlikely to be a practical design tool for three-dimensional problems in the foreseeable future.

\section{(c) Numerical implementation of Burton's method}

A promising alternative approach is to implement Burton's perturbation method numerically, leading to an eigenvalue problem to determine the stability boundary. If the exponential growth rate of the dominant perturbation can be assumed to be real, the critical sliding speed is defined by the condition that there exists a steadystate equilibrium perturbation, i.e. one with zero growth rate. Du et al. (1997) used the finite-element method to develop the matrix defining this eigenvalue problem. Yi et al. (1999) used Du et al.'s method to explore the effect of disc geometry in an idealized disc brake in which the brake pads are assumed to be rigid and nonconducting. Their results showed that the critical speed is in many cases quite close to that predicted by the considerably simpler analysis of Lee \& Barber (1993), which probably explains the success of that analysis in practical applications.

Du et al.'s method rests on the assumption that the dominant perturbation has a real growth rate. The limited range of problems that have been solved analytically suggest that this assumption is justified if one of the two sliding bodies is a thermal insulator, or if the dominant perturbation is independent of the coordinate in the sliding direction, as in 'banding' instabilities in axisymmetric systems, where the frictional heating is concentrated in an axisymmetric annular band within the contact area. However, a rigorous proof of this result has never been advanced. When both 
materials are thermally conducting, the stability boundary is generally determined by a disturbance that migrates with respect to both bodies in or opposed to the direction of sliding (Burton et al. 1973). In a stationary frame of reference, the perturbation will then appear to oscillate in time, corresponding to a complex exponential growth rate. The migration speed is smaller than for the better thermal conductor and this relative motion approaches zero when the other body tends to the limit of thermal insulation. Practical systems such as brakes and clutches usually involve a steel or cast-iron disc sliding against a composite friction material of significantly lower conductivity (typically two orders of magnitude lower than that of steel). As a result, the dominant perturbation migrates only very slowly relative to the metal disc. However, this migration plays an important part in the process, because it reduces the thermal expansion due to a given perturbation in heat input and hence increases the critical speed.

\section{(d) Eigenvalue formulation for the exponential growth rate}

Burton's method can be implemented numerically for systems of two thermal conductors by defining the eigenvalue problem for the exponential growth rate. This method was first suggested by Yeo \& Barber (1996), who developed it in the context of the related static thermoelastic contact problem, where instability results from the pressure dependence of an interfacial contact resistance.

We first assume that the temperature, stress and displacement fields can be written as the product of a function of the spatial coordinates $(x, y, z)$ and an exponential function of time; for example, the temperature field $T(x, y, z, t)$ is written

$$
T(x, y, z, t)=\mathrm{e}^{b t} \Theta(x, y, z) .
$$

When these expressions are substituted into the governing equations and boundary conditions of the problem, the exponential factor cancels and we are left with a modified system of equations in which the growth rate $b$ appears as a linear parameter. A finite-element discretization of this modified problem then yields a linear eigenvalue problem for $b$.

In order to adapt this method to the sliding contact problem, we need to choose a suitable frame of reference, relative to which at least one of the bodies will necessarily be moving. This introduces convective terms into the heat-conduction equation and can present numerical problems when the convective term is large (Christie et al. 1976). The relative magnitude of convective and diffusive terms can be assessed by calculating the Peclet number

$$
P e=V a / k,
$$

where $V$ is the convective velocity, $k$ is the thermal diffusivity, and $a$ is a representative length-scale. Peclet numbers in tribological applications are typically very large. For example, a steel clutch disc of mean diameter $0.2 \mathrm{~m}$ rotating at $2000 \mathrm{rpm}$ corresponds to $P e \approx 3.5 \times 10^{5}$ using the mean diameter for $a$, and even the element Peclet number will be large compared with unity for a realistic discretization. Thus, the convective terms will tend to dominate the finite-element solution.

Fortunately, difficulties with convective terms can be avoided by using Fourier reduction in the sliding direction as long as

(i) no material points on either sliding body experience intermittent contact, and 


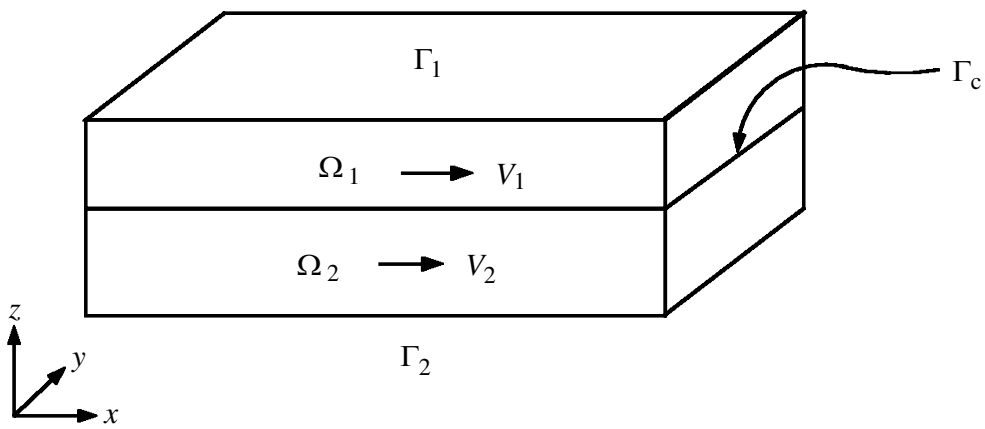

Figure 3. Schematic model for the two-body sliding system.

(ii) periodic boundary conditions apply in the sliding direction.

These conditions are satisfied for multi-disc brakes and clutches, which have an axisymmetric geometry, but which often exhibit signs of damage attributable to TEI with a non-axisymmetric eigenmode. In this case, orthogonality arguments show that all the eigenmodes must have Fourier form in the circumferential direction and the temperature field (for example) can be written in the form

$$
T(r, \phi, z, t)=\operatorname{Re}\left\{\mathrm{e}^{b t+\mathrm{j} n \phi} \Theta(r, z)\right\},
$$

where $(r, \phi, z)$ are cylindrical polar coordinates, $n$ is a wavenumber, and $\mathrm{j}=\sqrt{ }-1$. Once again, the (complex) exponential term cancels in the governing equations and the boundary conditions, leading to a reduced problem in $(r, z)$ only. A finite-element implementation of this reduced problem leads to a complex linear eigenvalue problem for $b$ for given values of $n$ and the sliding speed, $V$.

\section{Solution method}

It is convenient to consider first the related problem in three-dimensional Cartesian coordinates, since this permits a direct comparison with the results of Lee \& Barber (1993), which can therefore be used to validate the computational algorithm. The system is shown schematically in figure 3 . Two bodies, $\Omega_{1}, \Omega_{2}$, with boundaries $\Gamma_{1}$, $\Gamma_{2}$, respectively, slide in the positive $x$-direction at constant speeds $V_{1}, V_{2}$, respectively, and make contact over a common interface, $\Gamma_{\mathrm{c}}$, which is independent of time, $t$. The bodies are assumed to be of infinite extent in the $x$-direction and to have a cross-section that is independent of $x$, in which case the system will support solutions that are sinusoidal in $x$.

\section{(a) The heat-transfer problem}

The heat-conduction equation for body $\beta(\beta=1,2)$ in the fixed frame of reference $x, y, z$ is

$$
K_{\beta} \nabla^{2} T-\rho_{\beta} c_{\beta}\left(\frac{\partial T}{\partial t}+V_{\beta} \frac{\partial T}{\partial x}\right)=0,
$$

where $K_{\beta}, \rho_{\beta}, c_{\beta}$ are the thermal conductivity, density and specific heat, respectively, of material $\beta$. 
By analogy with equation (1.3), we assume an exponentially growing perturbation solution of the form

$$
T(x, y, z, t)=\operatorname{Re}\left\{\mathrm{e}^{b t+\mathrm{j} m x} \Theta(y, z)\right\},
$$

where $b$ is a possibly complex exponential growth rate and $m$ is a real wavenumber. $\dagger$ This function will satisfy equation (2.1) if

$$
K_{\beta} \nabla^{2} \Theta-\left[K_{\beta} m^{2}+\rho_{\beta} c_{\beta}\left(\mathrm{j} m V_{\beta}+b\right)\right] \Theta=0 .
$$

Notice that $\Theta$ is a function of $(y, z)$ only, so equation (2.3) defines a two-dimensional problem in the $y z$-plane. Appropriate boundary conditions on $\Theta$ for body $\beta(\beta=1,2)$ are

$$
K_{\beta} \frac{\partial T}{\partial n_{\beta}}=-q_{\beta} \quad \text { on } \Gamma_{\mathrm{c}}^{\beta}
$$

and

$$
K_{\beta} \frac{\partial T}{\partial n_{\beta}}=0 \quad \text { on } \Gamma_{\beta}-\Gamma_{\mathrm{c}}^{\beta},
$$

where $\Gamma_{\beta}$ is the boundary of body $\Omega_{\beta}, \Gamma_{\mathrm{c}}^{\beta}$ is that part of $\Gamma_{\beta}$ that is in contact, $n_{\beta}$ is the inward normal to $\Omega_{\beta}$ and $q_{\beta}$ is defined such that the heat flux into $\Omega_{\beta}$ at the contact surface is $\operatorname{Re}\left\{q_{\beta} \mathrm{e}^{b t+\mathrm{j} m x}\right\}$.

To obtain a finite-element formulation of the problem, we multiply the governing equation (2.3) by an arbitrary trial function, $v(y, z)$, and integrate over the domain $\Omega_{\beta}$, with the result

$$
\iint_{\Omega_{\beta}}\left\{K_{\beta} \nabla^{2} \Theta-\left[K_{\beta} m^{2}+\rho_{\beta} c_{\beta}\left(\mathrm{j} m V_{\beta}+b\right) \Theta\right]\right\} v \mathrm{~d} \Omega_{\beta}=0 .
$$

We then use the divergence theorem to integrate the first term by parts, obtaining, after some rearrangement,

$$
\begin{aligned}
& K_{\beta} \iint_{\Omega_{\beta}} \nabla v \cdot \nabla \Theta \mathrm{d} \Omega_{\beta}+\left[K_{\beta} m^{2}+\rho_{\beta} c_{\beta}\left(\mathrm{j} m V_{\beta}+b\right)\right] \\
& \times \iint_{\Omega_{\beta}} \Theta v \mathrm{~d} \Omega_{\beta}-K_{\beta} \int_{\Gamma_{\beta}} v \frac{\partial \Theta}{\partial n_{\beta}} \mathrm{d} \Gamma_{\beta}=0 .
\end{aligned}
$$

The last term in this equation can then be simplified using equations (2.4) and (2.5) with the result

$$
\begin{aligned}
K_{\beta} \iint_{\Omega_{\beta}} \nabla v \cdot \nabla \Theta \mathrm{d} \Omega_{\beta}+\left[K_{\beta} m^{2}\right. & \left.+\rho_{\beta} c_{\beta}\left(\mathrm{j} m V_{\beta}+b\right)\right] \\
& \times \iint_{\Omega_{\beta}} \Theta v \mathrm{~d} \Omega_{\beta}+\int_{\Gamma_{\mathrm{c}}^{\beta}} v q_{\beta} \mathrm{d} \Gamma_{\beta}=0 .
\end{aligned}
$$

$\dagger$ Notice that $m$ has the dimensions (length) ${ }^{-1}$ and can take all positive real values. By contrast, $n$ in equation (1.3) is dimensionless and must be a positive integer. 
We represent $\Theta$ in the discrete form

$$
\Theta(y, z)=\sum_{i=1}^{N_{\beta}} \Theta_{i} W_{i}(y, z),
$$

where $\Theta_{i}$ are the nodal values $\left(i=1, N_{\beta}\right)$, and $W_{i}$ is a set of $N_{\beta}$ shape functions.

There are no convective terms in the governing equation (2.8), since there is no discretization in the sliding direction. We can therefore safely use the Galerkin formulation in which the same functions $W_{i}$ are used as both shape and weight functions, leading to

$$
\begin{array}{r}
K_{\beta} \iint_{\Omega_{\beta}} \sum_{i=1}^{N_{\beta}} \Theta_{i}\left(\frac{\partial W_{j}}{\partial y} \frac{\partial W_{i}}{\partial y}+\frac{\partial W_{j}}{\partial z} \frac{\partial W_{i}}{\partial z}\right) \mathrm{d} \Omega_{\beta}+\left[K_{\beta} m^{2}+\rho_{\beta} c_{\beta}\left(\mathrm{j} m V_{\beta}+b\right)\right] \\
\times \iint_{\Omega_{\beta}} \sum_{i=1}^{N_{\beta}} \Theta_{i} W_{j} W_{i} \mathrm{~d} \Omega_{\beta}+Q_{j}^{\beta}=0, \quad j=1, N_{\beta},
\end{array}
$$

where

$$
Q_{j}^{\beta}=\int_{\Gamma_{\mathrm{c}}^{\beta}} W_{j} q_{\beta} \mathrm{d} \Gamma_{\beta}
$$

is the nodal heat source and is zero for all nodes except the contact nodes in $\Gamma_{\mathrm{c}}^{\beta}$.

The two sets of equations defined by (2.10) for $\beta=1,2$ can be combined into a single set. For this purpose, we note that the common contact nodes should appear only once, since we assume temperature continuity in the contact region. There are therefore $N=N_{1}+N_{2}-N_{\mathrm{c}}$ nodes in the combined domain $\Omega=\Omega_{1}+\Omega_{2}$, where $N_{\mathrm{c}}$ is the number of contact nodes. The corresponding heat sources in $\Gamma_{\mathrm{c}}$ should be added to define

$$
Q_{j}=Q_{j}^{1}+Q_{j}^{2},
$$

which are the nodal heat sources at the contact interface due to frictional heating. At all the other nodes in $\Omega$, there is no heat source $\left(Q_{j}=0\right)$.

We can write the resulting equations in the matrix form

$$
(\boldsymbol{K}+\boldsymbol{C}+b \boldsymbol{H}) \boldsymbol{\Theta}+\boldsymbol{Q}=0,
$$

where

$$
\boldsymbol{K}=\iint_{\Omega} K_{\beta}\left(\frac{\partial \boldsymbol{W}}{\partial y} \frac{\partial \boldsymbol{W}^{\mathrm{T}}}{\partial y}+\frac{\partial \boldsymbol{W}}{\partial z} \frac{\partial \boldsymbol{W}^{\mathrm{T}}}{\partial z}\right) \mathrm{d} \Omega
$$

is the conductivity matrix,

$$
\boldsymbol{H}=\iint_{\Omega} \rho_{\beta} c_{\beta} \boldsymbol{W} \boldsymbol{W}^{\mathrm{T}} \mathrm{d} \Omega
$$

is the mass matrix,

$$
\boldsymbol{C}=\iint_{\Omega}\left(K_{\beta} m^{2}+\mathrm{j} m \rho_{\beta} c_{\beta} V_{\beta}\right) \boldsymbol{W} \boldsymbol{W}^{\mathrm{T}} \mathrm{d} \Omega,
$$

and $\boldsymbol{W}=\left[W_{1}, \ldots, W_{N_{\beta}}\right]^{\mathrm{T}}$ is a column vector of shape functions. These matrices are easily evaluated using the four-node Gaussian quadrature method. 


\section{(b) The thermoelastic problem}

Equation (2.13) defines the temperature, $\boldsymbol{\Theta}$, due to a prescribed set of nodal heat sources, $\boldsymbol{Q}$. These heat sources are non-zero only at the contact interface, $\Gamma_{\mathrm{c}}$, and are the result of frictional heating. We denote this non-zero subset of nodal heat sources $\tilde{\boldsymbol{Q}}$ and they are given by

$$
\tilde{\boldsymbol{Q}}=f V \tilde{\boldsymbol{P}},
$$

where $\tilde{\boldsymbol{P}}$ is the corresponding vector of nodal contact forces normal to the contact interface, $f$ is the coefficient of friction, and $V=\left|V_{1}-V_{2}\right|$ is the relative sliding velocity.

To complete the solution of the thermomechanically coupled problem, we need to solve a thermoelastic contact problem to determine the linear relation between the nodal forces, $\tilde{\boldsymbol{P}}$, and the nodal temperatures, $\boldsymbol{\Theta}$. This relation can be written in the symbolic form

$$
\tilde{P}_{i}=\sum_{j=1}^{N} A_{i j} \Theta_{j}, \quad i=1, N_{\mathrm{c}},
$$

where $A_{i j}$ is a non-square matrix of order $N_{\mathrm{c}} \times N$.

We seek to determine the matrix $\boldsymbol{A}$, which constitutes the general solution of the thermoelastic problem. It is somewhat more straightforward than the heatconduction problem, since there are no time-dependent terms in the quasi-static thermoelastic governing equations, so the only modification to these equations is that introduced by the Fourier spatial variation $\exp (\mathrm{j} m x)$. In particular, the resulting matrix $\boldsymbol{A}$ has no dependence on the velocities $V_{\beta}$ or the exponential growth rate $b$.

The most computationally efficient procedure for determining $\boldsymbol{A}$ is to write a custom-made finite-element solution of the thermoelastic problem in which the Fourier term, $\exp (\mathrm{j} m x)$, is cancelled before discretization (as in $\S 2 a$ above). This method was described by Yeo \& Barber (1996) and it permits $\boldsymbol{A}$ to be determined from a single finite-element run for each Fourier term.

An alternative method is to use a commercial code to determine the nodal force vector $\tilde{\boldsymbol{P}}^{(j)}$ due to the nodal temperatures $\Theta_{k}=\delta_{k j}$, where $\delta_{k j}$ is the Kronecker delta. Substitution in equation (2.18) then gives

$$
A_{i j}=\tilde{P}_{i}^{(j)}, \quad i=1, N_{\mathrm{c}},
$$

which determines the $j$ th column of $\boldsymbol{A}$. The complete matrix can be determined column by column from a series of such runs for $j=1, N$. This method requires $N$ finiteelement runs for each Fourier term, but it involves less initial programming effort, most of which can be performed within the user-friendly environment of the commercial code. Many commercial codes do not contain appropriate Fourier elements, particularly in Cartesian coordinates, but these can often be defined with a 'user element' option. The essential mathematics for this purpose is given in Appendix A, for the axisymmetric geometry. An alternative strategy is to use a three-dimensional finiteelement model and impose the above conditions in the form $T_{k}(x)=\delta_{k j} \cos (m x)$. The corresponding value of $\tilde{\boldsymbol{P}}^{(j)}$ can then be obtained by Fourier inversion of the variation of the resulting nodal forces in the $x$-direction. 
In the present study we experimented with all of these methods, all of which gave similar results. Yeo \& Barber's (1996) method is by far the most efficient and is worth the extra programming effort for anyone wishing to use the method on a regular basis. However, for a limited number of calculations, familiarity with the commercial software may outweigh the slower solution procedure.

\section{(c) Development of the eigenvalue equation}

Once the matrix $\boldsymbol{A}$ has been determined, we can eliminate $\tilde{\boldsymbol{P}}$ between equations (2.17) and (2.18), obtaining

$$
\tilde{\boldsymbol{Q}}=f V \boldsymbol{A} \Theta .
$$

Notice that $\tilde{\boldsymbol{Q}}$ is defined only at the $N_{\text {c }}$ contact nodes, whereas the nodal heat source vector, $\boldsymbol{Q}$, in equation $(2.13)$ is defined at all the $N$ nodes in $\Omega$. The two vectors are related by the equation

$$
Q=\Phi \tilde{Q},
$$

where the $N \times N_{\mathrm{c}}$ matrix $\boldsymbol{\Phi}$ is defined by

$$
\boldsymbol{\Phi}=\left[\begin{array}{l}
\boldsymbol{I} \\
0
\end{array}\right]
$$

and $\boldsymbol{I}$ is the identity matrix of order $N_{\mathrm{c}} \times N_{\mathrm{c}}$. Multiplying (2.20) by $\boldsymbol{\Phi}$ and using (2.21), we obtain the $N \times N$ matrix equation

$$
\boldsymbol{Q}=f V \boldsymbol{\Phi} \boldsymbol{A} \boldsymbol{\Theta}
$$

Finally, substituting for $\boldsymbol{Q}$ from (2.13) and rearranging, we obtain

$$
[(\boldsymbol{K}+\boldsymbol{C}+f V \boldsymbol{\Phi} \boldsymbol{A})+b \boldsymbol{H}] \boldsymbol{\Theta}=0,
$$

which is an $N \times N$ linear eigenvalue problem for the exponential growth rate, $b$. The resulting eigenvalue depends on the sliding speed, $V$, and also on the Fourier number, $m$, since this appears in the definition of the matrix, $\boldsymbol{C}$. We also note that $\boldsymbol{C}$ is a complex matrix, so that the eigenvalues and eigenvectors are generally complex.

\section{(d) Axisymmetric geometries}

The same method is easily extended to the stability of Fourier perturbations in the sliding of axisymmetric bodies. Axisymmetric or almost axisymmetric geometries arise in automotive transmission clutches and in multi-disc aircraft brakes and are therefore of considerable technological importance, particularly since TEI often places serious limitations on the performance of these components.

The polar coordinate equivalent of equation (2.1) is

$$
K_{\beta}\left(\frac{\partial^{2} T}{\partial r^{2}}+\frac{1}{r} \frac{\partial T}{\partial r}+\frac{1}{r^{2}} \frac{\partial^{2} T}{\partial \phi^{2}}+\frac{\partial^{2} T}{\partial z^{2}}\right)-\rho_{\beta} c_{\beta}\left(\frac{\partial T}{\partial t}+\omega_{\beta} \frac{\partial T}{\partial \phi}\right)=0,
$$

where $\omega_{\beta}$ is the angular velocity of body $\beta$ in the chosen frame of reference. As before, we substitute the form (1.3) for $T(r, \phi, z, t)$, obtaining

$$
K_{\beta}\left(\frac{\partial^{2} \Theta}{\partial r^{2}}+\frac{1}{r} \frac{\partial \Theta}{\partial r}+\frac{\partial^{2} \Theta}{\partial z^{2}}\right)-\left[\frac{K_{\beta} n^{2}}{r^{2}}+\rho_{\beta} c_{\beta}\left(\mathrm{j} n \omega_{\beta}+b\right)\right] \Theta=0 .
$$


Notice that the unknown temperature function, $\Theta(r, z)$, is defined only in the twodimensional spatial domain, $\Omega_{\beta}$, representing the cross-section of the axisymmetric geometry at constant $\phi$.

The finite-element solution of the heat-conduction problem now proceeds exactly as in $\S 2 a$, leading to the same final equation (2.13), but with the modified matrices

$$
\begin{aligned}
\boldsymbol{K} & =\iint_{\Omega} K_{\beta}\left(\frac{\partial \boldsymbol{W}}{\partial r} \frac{\partial \boldsymbol{W}^{\mathrm{T}}}{\partial r}-\frac{\boldsymbol{W}}{r} \frac{\partial \boldsymbol{W}^{\mathrm{T}}}{\partial r}+\frac{\partial \boldsymbol{W}}{\partial z} \frac{\partial \boldsymbol{W}^{\mathrm{T}}}{\partial z}\right) \mathrm{d} \Omega, \\
\boldsymbol{H} & =\iint_{\Omega} \rho_{\beta} c_{\beta} \boldsymbol{W} \boldsymbol{W}^{\mathrm{T}} \mathrm{d} \Omega, \\
\boldsymbol{C} & =\iint_{\Omega}\left(\frac{K_{\beta} n^{2}}{r^{2}}+\mathrm{j} n \rho_{\beta} c_{\beta} \omega_{\beta}\right) \boldsymbol{W} \boldsymbol{W}^{\mathrm{T}} \mathrm{d} \Omega .
\end{aligned}
$$

In the axisymmetric geometry, the relative sliding speed varies with radius, being given by

$$
V=\omega r
$$

where $\omega=\left|\omega_{1}-\omega_{2}\right|$ is the relative angular velocity. The nodal heat sources, $\tilde{\boldsymbol{Q}}$, at the $N_{\mathrm{c}}$ contact nodes are, therefore, given by

$$
\tilde{\boldsymbol{Q}}=f \boldsymbol{V} \tilde{\boldsymbol{P}}
$$

where $\boldsymbol{V}$ is the diagonal $N_{\mathrm{c}} \times N_{\mathrm{c}}$ matrix defined by

$$
V_{j i}=\omega r_{i} \delta_{j i}
$$

and $r_{i}$ is the radial coordinate of the $i$ th contact node.

The matrix $\boldsymbol{A}$ is determined as in $\S 2 b$ and the solution of the coupled problem results in the $N \times N$ linear eigenvalue problem

$$
[(\boldsymbol{K}+\boldsymbol{C}+f(\boldsymbol{V} \boldsymbol{\Phi}) \boldsymbol{A})+b \boldsymbol{H}] \boldsymbol{\Theta}=0,
$$

for the exponential growth rate, $b$.

\section{Results and discussion}

We first apply the method to the two-dimensional problem solved analytically by Lee $\&$ Barber (1993). This will allow us to validate the method and also to explore the mesh refinement required to give a good description of the dominant eigenfunction and the critical speed. For this purpose, we used the properties of cast iron for the layer and of a commercial friction material for the half-planes. The appropriate physical properties $\dagger$ are tabulated in table 1 and correspond to typical automotive disc brake practice.

$\dagger$ We assume here that the friction material is isotropic and that the properties are independent of mean temperature. Typical friction materials are significantly anisotropic, but manufacturers generally only quote values of the modulus for compression across the thickness. If more complete constitutive data are available, they are easily incorporated into the solution using equations (A 5) and (A 6) of Appendix A. The thermal expansion coefficient for many friction materials increases significantly with mean temperature, and this can cause systems to become unstable at later stages in a braking event (Lee \& Barber 1994). 
Table 1. Material properties for the layer problem

\begin{tabular}{lcc}
\hline & layer & half-plane \\
\hline Young's modulus, $E(\mathrm{GPa})$ & 125 & 0.53 \\
Poisson's ratio, $\nu$ & 0.25 & 0.25 \\
thermal expansion coefficient, $\alpha\left({ }^{\circ} \mathrm{C}^{-1}\right)$ & $12 \times 10^{-6}$ & $30 \times 10^{-6}$ \\
thermal conductivity, $K\left(\mathrm{~W} \mathrm{~m}{ }^{-1}{ }^{\circ} \mathrm{C}^{-1}\right)$ & 54.0 & 0.5 \\
thermal diffusivity, $k\left(\mathrm{~mm}^{2} \mathrm{~s}^{-1}\right)$ & 13.0 & 0.27 \\
\hline
\end{tabular}

The friction coefficient was taken as $f=0.4$ and the layer semi-thickness as $a=$ $10 \mathrm{~mm}$. The half-planes were modelled as layers of finite thickness equal to $900 \mathrm{~mm}$. This is about three times larger than the dominant wavelength for the problem, which is sufficiently large to ensure that the distant boundary has no effect on the perturbation problem. The system is symmetric about the mid-plane of the layer and all the modes are either symmetric or antisymmetric. We therefore modelled half of the system (half of the layer and one half-plane) and used antisymmetric boundary conditions at the symmetry plane, since Lee \& Barber (1994) showed that the antisymmetric mode is always dominant for practical material combinations.

\section{(a) Meshing and convergence}

Fourier reduction converts the layer/half-plane problem into a one-dimensional problem and hence only meshing in the direction normal to the interface needs to be considered. As explained in $\S 1 b$ above, the disturbance moves relatively slowly over the good conductor (the layer) but with a speed close to the sliding speed over the half-plane. The Peclet number for the half-plane is, therefore, extremely high, and as a result the thermal disturbance is concentrated in a very small boundary layer near the contact surface. It is essential to use a fine mesh in this region in order to describe the temperature field adequately. However, the related elastic problem is less localized, with significant stress variation at depths comparable with the wavelength of the disturbance.

In order to capture these two processes with dissimilar length-scales, a graded mesh was used with a bias ratio (i.e. the ratio between the lengths of adjacent elements) of 0.7 . In this way, a mesh with only 20 elements in the half-plane has a smallest element (at the surface) of dimension less than $0.04 \%$ of the total depth modelled.

The degree of mesh refinement required near the critical speed can be estimated by considering the heat-conduction problem in which a constant Fourier disturbance moves over the surface of a half-plane at speed, $c_{1}$. Elementary calculations show that the resulting thermal disturbance decays exponentially with depth according to

$$
\Theta(y)=\Theta_{0} \exp (-\lambda y)
$$

where the complex decay rate

$$
\lambda=\sqrt{m^{2}-\frac{\mathrm{j} c_{1} m}{k}} .
$$

If the migration Peclet number is large, i.e.

$$
P e=\frac{c_{1}}{k m} \gg 1
$$




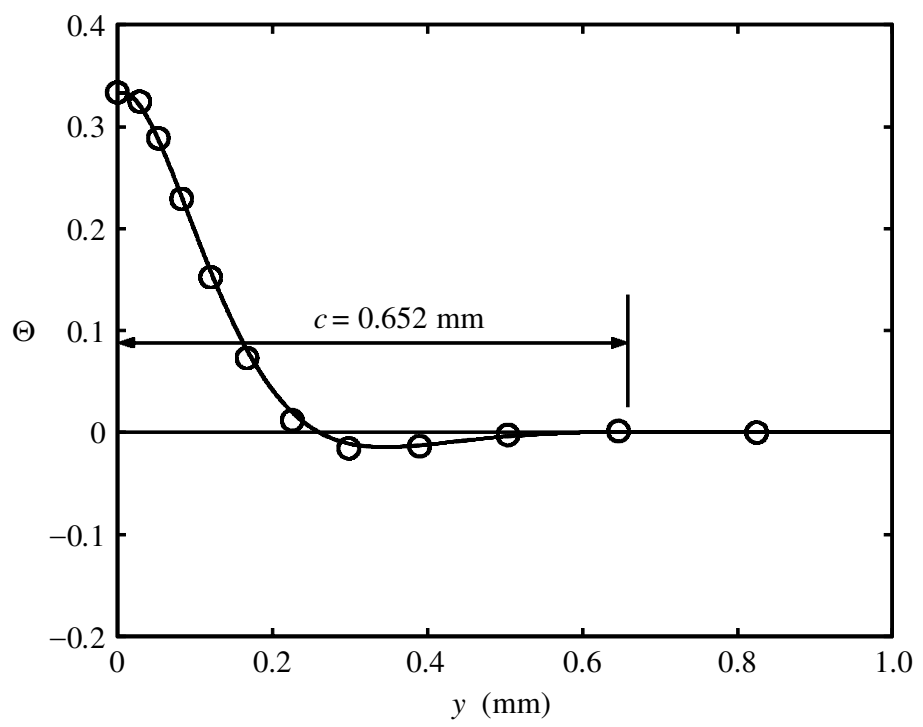

Figure 4. Comparison of the analytical solution and the finite-element results for $\Theta(y)$ (o, finite-element solution; ——, analytical solution).

this expression can be approximated by

$$
\lambda=(1-\mathrm{j}) \sqrt{\frac{V m}{2 k}},
$$

where we have used the fact that $c_{1} \approx V$ for the poor conductor. This represents an oscillatory decaying disturbance of wavelength

$$
\chi=2 \pi \sqrt{\frac{2 k}{V m}} .
$$

Equations (3.1) and (3.4) show that $\Theta(y)$ decays to less than $0.2 \%$ of $\Theta_{0}$ within one wavelength, and hence to capture the thermal disturbance it is essential to use a reasonable number of elements in the domain $0<y<\chi$. Figure 4 compares the theoretical temperature function $\Theta(y)$ at the critical speed from Lee \& Barber's (1993) analysis with the corresponding finite-element results, using a mesh with 30 elements and a bias ratio of 0.7 , which provides 10 elements in the first wavelength, $0<y<\chi$. The agreement is extremely good, showing that the above strategy is successful in determining the degree of mesh refinement required to capture the thermal boundary-layer effect. An alternative strategy here would be to partition the poor conductor into two parts, using a fine mesh in the thermal boundary layer and a coarser mesh in the rest of the body.

For the materials used in this study, this strategy is required only in the poor conductor. Migration speeds in the good conductor were always sufficiently small to give thermal boundary-layer thicknesses comparable with the other system dimensions.

\section{(b) Critical speed and migration speed}

The finite-element formulation was used to obtain the complex eigenvalue, $b$, for various sliding speeds, $V$, and iteration was used to determine the critical speed, $V_{0}$, 


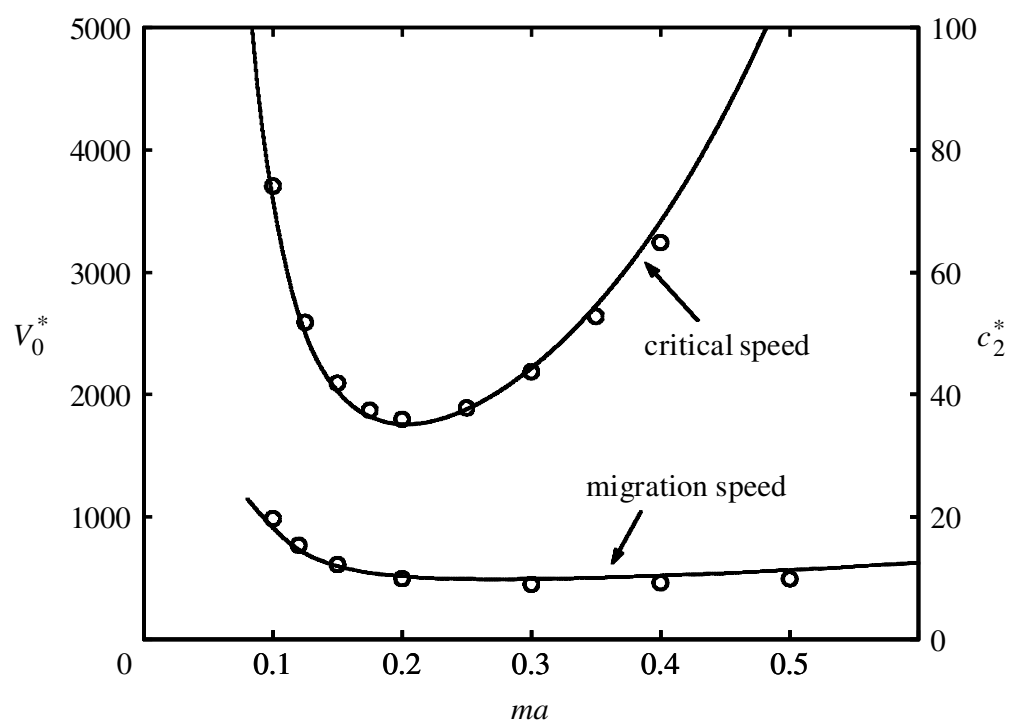

Figure 5. Comparison of the finite-element solution with the analytical solution of Lee \& Barber (1993).

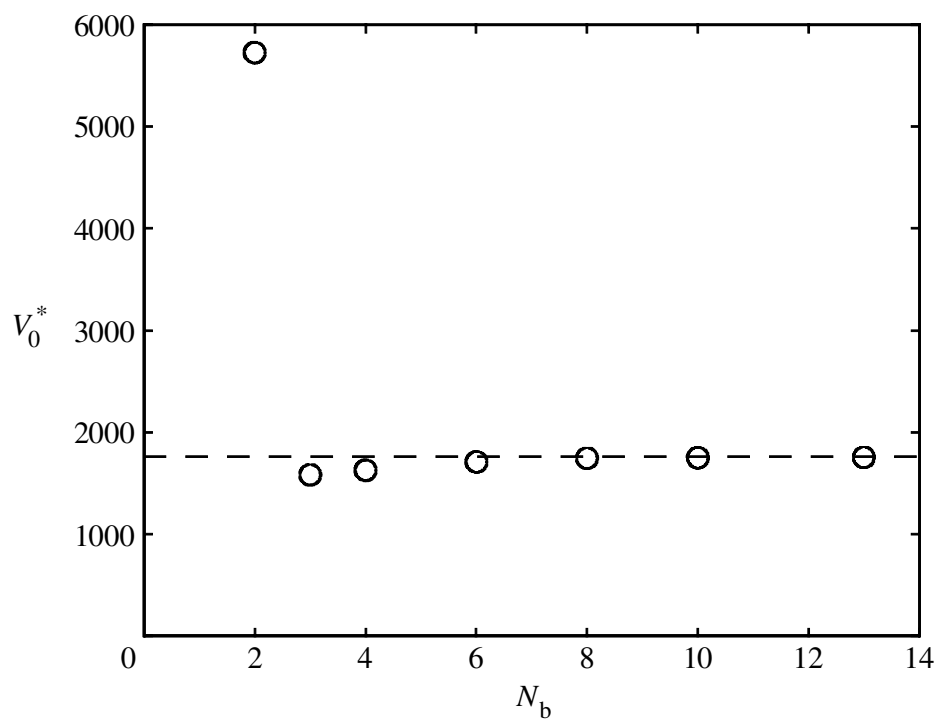

Figure 6. Sensitivity of the critical speed to the number of elements, $N_{\mathrm{b}}$, in the boundary layer (o, finite-element solution; ---, analytical solution).

at which the real part of the first eigenvalue reaches zero. Figure 5 shows the dimensionless critical speed, $V_{0}^{*}=V_{0} a / k_{2}$, as a function of dimensionless wavenumber $m a$, where $k_{2}$ is the thermal diffusivity of the cast-iron layer. The solid line represents the analytical solution of Lee \& Barber (1993) and the circles were obtained using the present finite-element method with 22 elements in the half-plane. Extremely good agreement was achieved throughout the critical range of wavenumber, $m$, confirming the effectiveness of the method for this category of problem. 
The imaginary part of the eigenvalue, $\operatorname{Im}(b)=c_{2}$, represents the migration velocity of the perturbation through the reference frame, which we fixed in the material of the layer. This migration velocity tends to zero in the limiting case, where the thermal conductivity of the moving material tends to zero and is small compared with the sliding speed for practical friction material/metal material pairs. The dimensionless migration speed $c_{2}^{*}=c_{2} a / k_{2}$ is compared with the predictions of Lee \& Barber (1993) in figure 5 and again the agreement is very good.

Figure 6 explores the sensitivity of the critical speed to the number of elements, $N_{\mathrm{b}}$, in the thermal boundary layer, defined as $0<y<\chi$, where $\chi$ is given by equation (3.5). Convergence on the exact result is non-monotonic because of the oscillatory nature of the temperature field presented in figure 4. Good results are obtained for $N_{\mathrm{b}} \geqslant 6$, but if $N_{\mathrm{b}}$ is too small, very large non-conservative errors can be obtained (i.e. the critical speed is very much overestimated). For example, with $N_{\mathrm{b}}=2$, the critical speed was overestimated by more than a factor of three, even though the smallest element in this case was only an apparently respectable $1.7 \%$ of the layer thickness, $2 a$. This highlights the danger of using numerical methods in such problems without adequate regard to thermal boundary-layer effects.

The thickness of the boundary layer (equation (3.5)) depends on the wavenumber $m$, and in general the dominant wavenumber is not known until the analysis is completed. This difficulty can be overcome by using a relatively coarse mesh to estimate the dominant wavenumber, which is then used to determine the mesh refinement required for a more accurate calculation.

\section{(c) Finite pad thickness}

In practical brakes and clutches, the pad is also of finite thickness and is supported by a more rigid backing plate. This increases the effective pad stiffness and generally lowers the critical speed. Hartsock \& Fash (1999) developed an approximate analytical solution to this problem. The basis of their method is the observation that the thermal boundary layer in the friction material is so small that the temperature field is essentially uninfluenced by the thermal boundary condition at the backing plate. Lee \& Barber's (1993) half-plane assumption can therefore be retained for the heat-conduction problem, and the influence of the finite thickness is felt only in the elastic problem, which defines the relation between the amplitude of a Fourier term in the contact pressure and that of the corresponding term in the normal surface displacement. Hartsock \& Fash (2000) solved this elementary elasticity problem and used their results to define a wavenumber-dependent 'effective modulus' that a halfplane would need to have to exhibit the same elastic behaviour. They then used this modulus in place of the real modulus in Lee \& Barber's (1993) solution.

The present method enables us to test Hartsock \& Fash's (2000) approximation numerically. Figure 7 shows the critical speed for a three-layer system comprising a central steel layer of thickness $2.75 \mathrm{~mm}$ and two friction material layers each of thickness $0.673 \mathrm{~mm}$, assumed to be rigidly supported at the non-contacting interface. The friction material properties were those of 'friction material 1 ' in table 2 and are based on transmission clutch practice. The experimentally measured friction coefficient for this material combination is $f=0.12$.

The solid line in figure 7 represents the analytical solution from Hartsock \& Fash (2000), while the points are the present finite-element results. The agreement is 
Table 2. Material properties for the three-layer and multi-disc clutch problems

\begin{tabular}{lccc}
\hline & steel & $\begin{array}{c}\text { friction } \\
\text { material 1 }\end{array}$ & $\begin{array}{c}\text { friction } \\
\text { material 2 }\end{array}$ \\
\hline Young's modulus, $E(\mathrm{GPa})$ & 200 & 0.11 & 0.30 \\
Poisson's ratio, $\nu$ & 0.30 & 0.25 & 0.12 \\
thermal expansion coefficient, $\alpha\left({ }^{\circ} \mathrm{C}^{-1}\right)$ & $12 \times 10^{-6}$ & $14 \times 10^{-6}$ & $14 \times 10^{-6}$ \\
thermal conductivity, $K\left(\mathrm{~W} \mathrm{~m}{ }^{-1}{ }^{\circ} \mathrm{C}^{-1}\right)$ & 42.0 & 0.22 & 0.241 \\
thermal diffusivity, $k\left(\mathrm{~mm}^{2} \mathrm{~s}^{-1}\right)$ & 11.9 & 0.122 & 0.177 \\
\hline
\end{tabular}

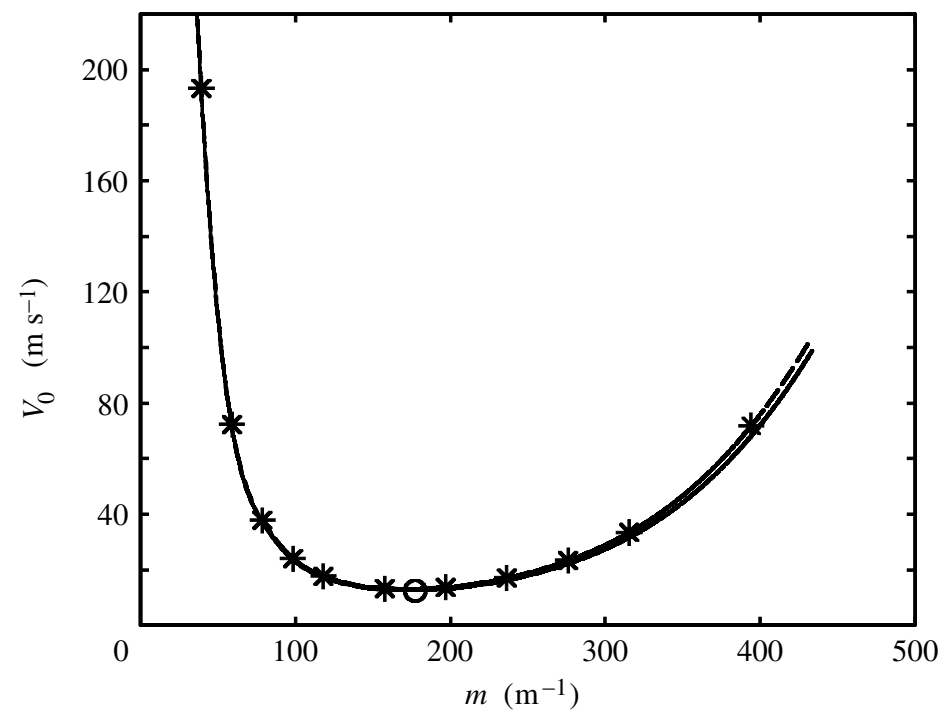

Figure 7. Critical speed for the two-dimensional three-layer model as a function of wavenumber, $m$ (*, two-dimensional finite-element model; —_, Hartsock \& Fash's (2000) model; o, Zagrodzki et al.'s (1999) simulation).

excellent, showing that the approximate representation of the temperature field is justified even for such a thin friction material layer. This holds out promise for the development of analytical models for more complex multi-disc brake and clutch applications.

Also shown in figure 7 is a single point representing the critical speed and wavenumber obtained for this system by Zagrodzki et al. (1999), using a full time domain simulation. Once again, this point agrees almost exactly with the finite-element and the approximate analytical results.

\section{(d) The axisymmetric geometry}

Practical brakes and clutches use axisymmetric annular discs of finite thickness and finite inner and outer radii. We first tested a simple geometry of this kind involving a single steel disc of thickness $2.75 \mathrm{~mm}$ sliding between two stationary friction material discs of thickness $0.673 \mathrm{~mm}$ with rigid backing plates. All three discs were taken to have inner radius $44.5 \mathrm{~mm}$ and outer radius $57.0 \mathrm{~mm}$. The friction material properties 


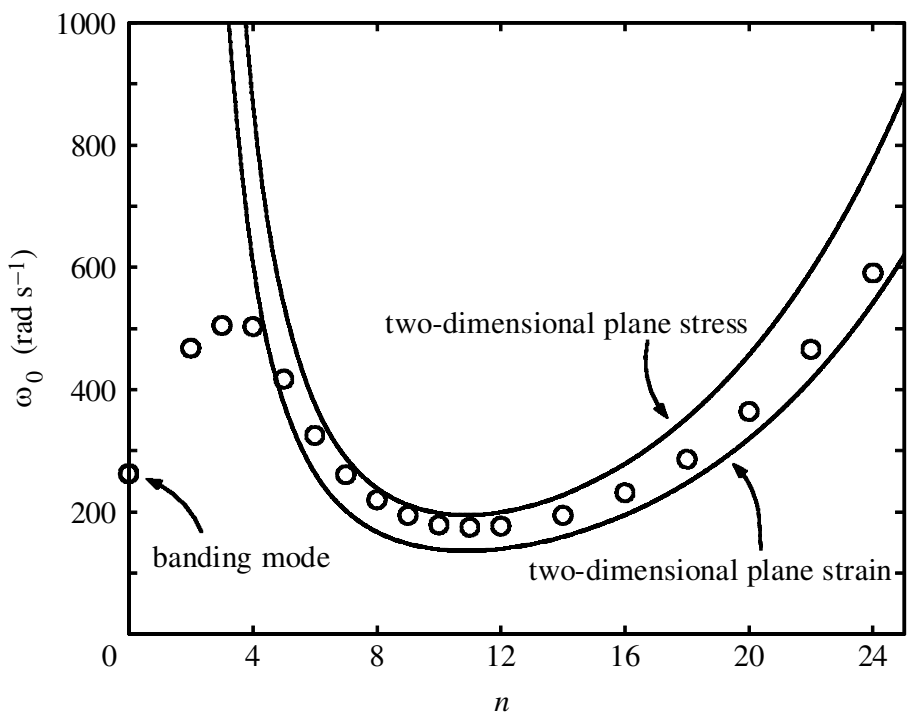

Figure 8. Critical rotational speed, $\omega_{0}$, for the three-layer axisymmetric clutch model (o, three-dimensional finite-element model; __ , Hartsock \& Fash's (2000) model).

were those of 'friction material 2' in table 2, for which the experimentally measured friction coefficient $f=0.13$.

Systems with axisymmetric geometry can only exhibit modes with an integer number of waves, $n$, around the circumference, including the special case $n=0$ corresponding to axisymmetric or 'banding' modes. Yi et al. (1999) showed that for a single disc sliding between two non-conducting rigid surfaces, the critical speed for sufficiently large wavenumber is bounded by the two-dimensional plane-strain and plane-stress solutions. A similar comparison is performed for the present two-material three-disc system in figure 8 . The solid lines represent predictions of the critical speed from the two-dimensional solution of Hartsock \& Fash (2000), while the results of the present finite-element solution are represented by os.

Stability of the system is determined by the wavenumber with lowest critical speed, which here corresponds to $n=11$. The three-dimensional results lie between the twodimensional plane-strain and plane-stress bounds for $n \geqslant 4$. The modes for $n<4$ involve several reversals of temperature through the radial thickness and these modes cannot of course be captured by a two-dimensional analysis. In particular, the critical speed for the first banding mode $(n=0)$ is fairly low, so that banding as well as focal modes might be expected with this geometry.

Additional runs were made to explore the sensitivity of the results to modest changes in the coefficient of friction. For example, increasing $f$ from 0.13 to 0.16 left the dominant eigenfunction (the number of hot spots) unchanged, but reduced the critical speed from $175 \mathrm{rad} \mathrm{s}^{-1}$ to $134 \mathrm{rad} \mathrm{s}^{-1}$.

\section{(e) A multi-disc clutch}

Finally, we apply the finite-element method to the practical clutch design shown schematically in figure 9 . The design parameters and material properties were chosen to be consistent with the clutch whose experimentally damaged discs are shown 


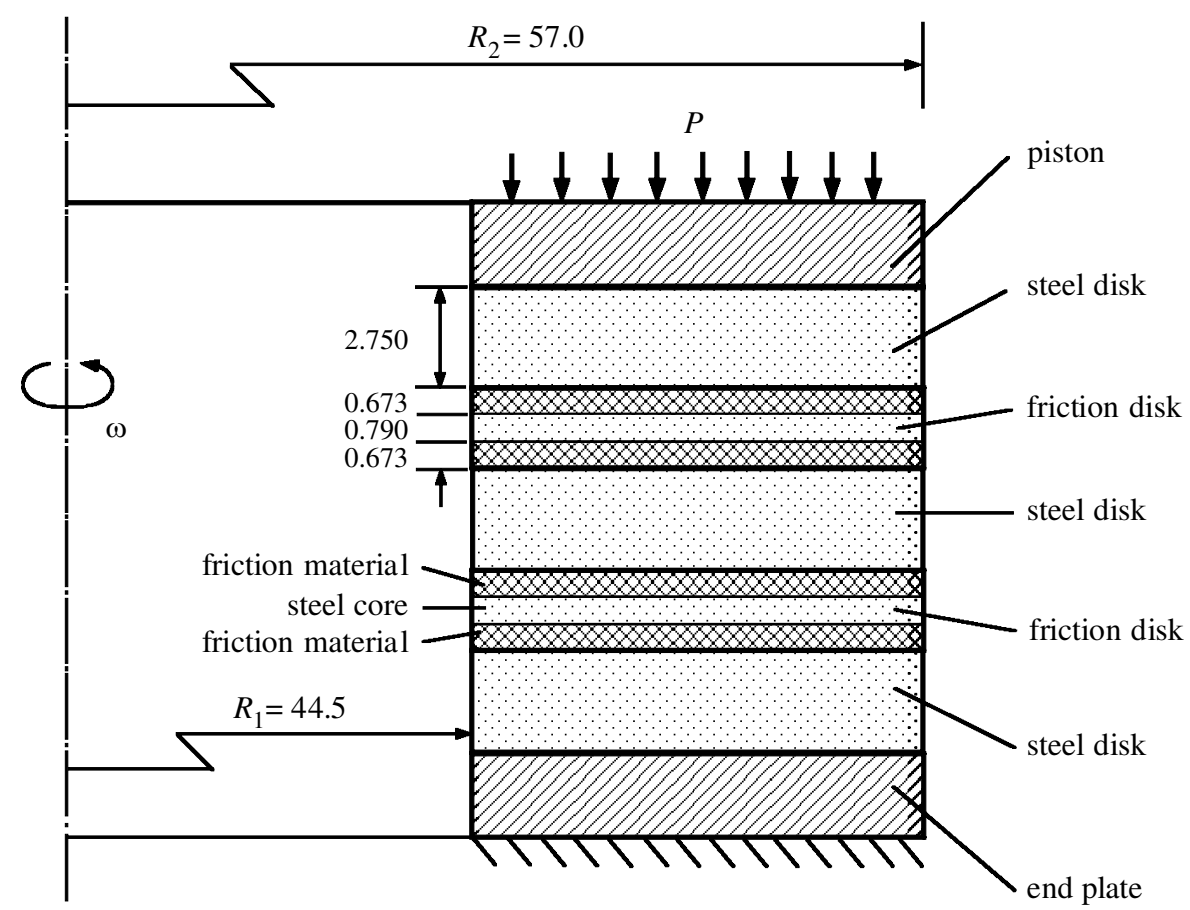

Figure 9. Typical multi-disc clutch system. All dimensions are in mm.

in figures 1 and 2. The clutch has three steel stators and two composite rotors. The rotors each have a steel core and a friction material layer bonded onto each side. During operation, sliding occurs between the friction material layers and the adjacent stators. A hydraulic pressure, $P$, is applied to the upper piston, causing the stack of discs to be compressed against the lower reaction plate. The corresponding boundary conditions on the (homogeneous) perturbation problem are therefore that the upper surface of the piston be traction free and the lower surface of the end plate be restrained against axial motion. All exposed surfaces were assumed to be thermally insulated, since practical heat-transfer coefficients are so small that they hardly affect thermoelastic instability. The material properties for this example are those of friction material 2 in table 2 above, for which the friction coefficient is again $f=0.13$.

The critical speed for the five-disc clutch system is shown as a function of wavenumber $n$ in figure 10. Instability first occurs in the banding mode $(n=0)$ at a rotational speed of $218 \mathrm{rad} \mathrm{s}^{-1}$, but there is also a local minimum of $237 \mathrm{rad} \mathrm{s}^{-1}$ at $n=10$. This clutch is designed for initial engagement speeds of $628 \mathrm{rad} \mathrm{s}^{-1}$, so we also calculated the exponential growth rate at this speed. The maximum value of $b=39.6 \mathrm{~s}^{-1}$ corresponds to the Fourier mode with 10 hot spots per revolution, while the banding mode grows only at a rate of $12.2 \mathrm{~s}^{-1}$. Thus, the non-axisymmetric mode would be expected to be dominant in this application. The eigenfunction for temperature in the stator surface is shown in figure 11 for the $n=10$ mode and the corresponding temperature contours in the $(r, z)$-plane are shown in figure 12 . Notice that the eigenmode is antisymmetric with respect to the central stator, in which the greatest 


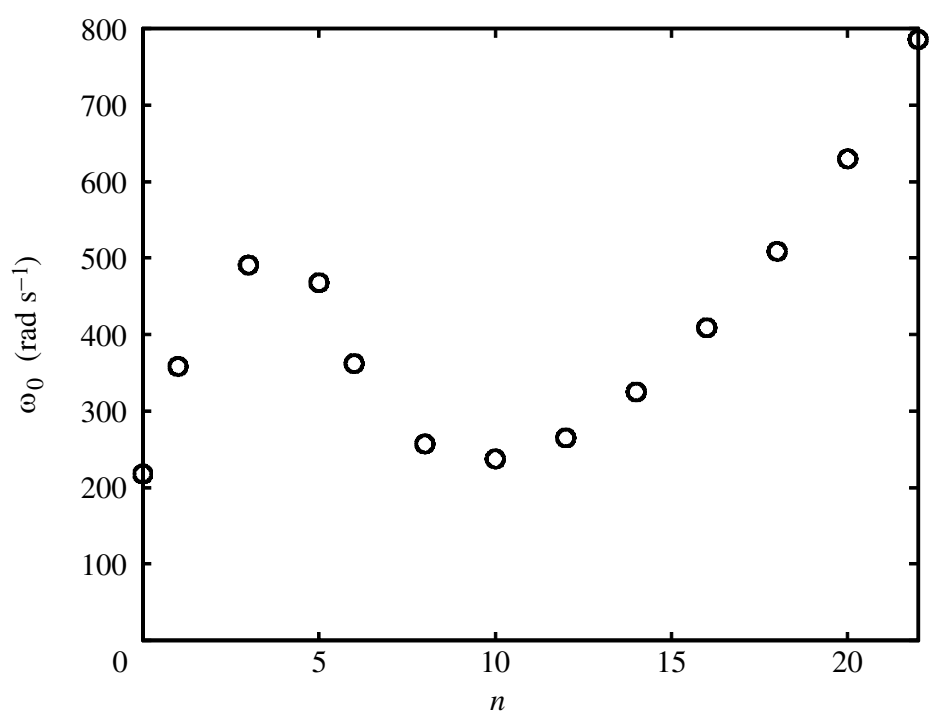

Figure 10. Critical speed as a function of wavenumber $n$ for the five-disc clutch.

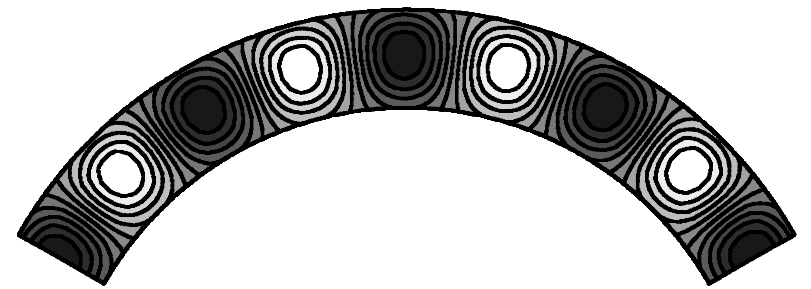

Figure 11. Dominant eigenmode for the temperature in the stator surface.

temperature perturbations are recorded. The temperatures in the rotors are close to zero except in a thermal boundary layer that is too thin to be visible in figure 12 .

Comparison of figures 11 and 12 with the experimental discs of figures 1 and 2 shows that the perturbation analysis correctly predicts an antisymmetric mode with focal hot spots, but the dominant wavenumber is predicted to be 10 , in contrast to the 12 hot spots observed experimentally. Various explanations might be advanced for this relatively small discrepancy. The initial speed for clutch engagement is well above the predicted critical value and all wavenumbers between 4 and 14 are unstable at the beginning of the engagement. However, the mode of figures 11 and 12 has the highest growth rate and would be expected to dominate the transient process. A more plausible explanation is that clutch friction materials exhibit quite complex constitutive behaviour and it is difficult to select an appropriate incremental elastic modulus for the analysis. The modulus given in table 2 is the incremental modulus obtained in compression tests at the mean engagement pressure, but significant stiffening may occur under service conditions. The critical speed and the dominant eigenmode are both quite sensitive to the modulus of the friction material and plausible values could have been chosen to 'fit' the theoretical predictions to a wavenumber of 12 . This highlights the fact that the principal difficulty remaining in obtaining reliable theoretical predictions for TEI performance lies in the accurate characterization of the properties of the complex friction materials used. 


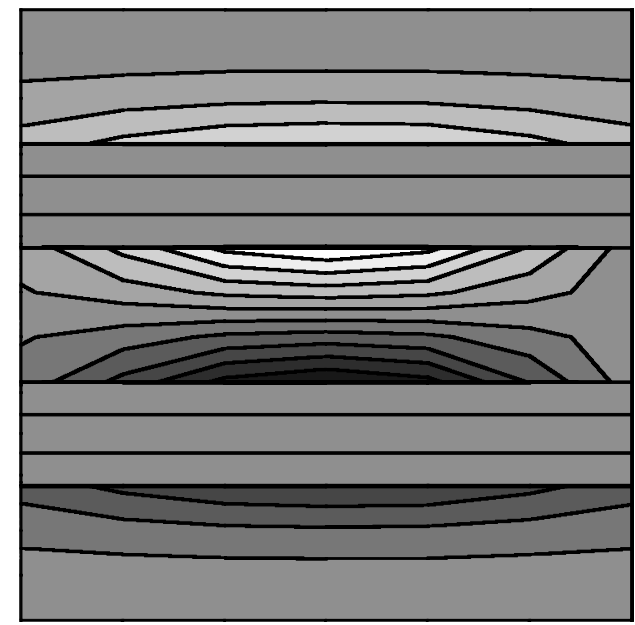

Figure 12. Temperature contours in the $(r, z)$-plane for the eigenmode of figure 10.

In figure 12, the two rotors exhibit a 'qualitative' symmetry, in the sense that hot regions occur at the same locations on the two sides, but the maximum temperatures are lower on those surfaces nearest to the piston and the end plate. Both these predictions were confirmed by the experimental observations. The most severe damage was observed on the central steel disc and the location of hot spots on the other two stators indicated a mode symmetric with respect to the rotors. The attenuation of the disturbance near the ends of the disc stack is probably attributable to the extra rigidity provided to the terminal stators by the piston and end plate. In fact, a simpler model in which the piston and end plate were replaced by rigid non-conducting surfaces predicted a critical speed within $1 \%$ of the more exact value.

This explanation also suggests that a more exact sequence of antisymmetric and symmetric perturbations in the stators and rotors, respectively, would be observed in a clutch with a larger number of discs. This was confirmed by additional finiteelement calculations for clutches of the same form as figure 9, but with odd numbers of discs between 3 and 13. The critical speed decreases towards a limit as the number of discs increases, and the dominant mode approaches a state in which the perturbation is strictly antisymmetric in the steel discs and symmetric in the composite discs, except for those near to the ends of the stack. This limiting condition was also obtained independently by modelling half of one rotor and one stator, using symmetric/antisymmetric boundary conditions at the respective mid-planes. The number of hot spots in the dominant eigenmode increases slightly with the number of discs, but the solution has essentially converged on the limit for clutches with 11 discs or more.

Notice that the axisymmetric mode in this example has the lowest critical speed but that a non-axisymmetric mode becomes dominant at larger speeds. The general solution of the transient thermoelastic contact problem at constant sliding speed can be written down as an eigenfunction expansion, i.e. as a series containing all possible terms of the form of equation (1.3). Furthermore, the fact that some of these terms grow exponentially but that most decay suggests that a severely truncated series (a reduced-order model) might give a highly efficient numerical approximation to the 
transient behaviour, while retaining adequate accuracy. This is the subject of an ongoing investigation.

\section{Conclusions}

The Fourier reduction method developed in this paper permits a remarkably efficient solution of the frictional thermoelastic stability problem for systems in which the geometry is axisymmetric. The power of the method is demonstrated by the multi-disc clutch example, direct numerical simulation of which would represent an extremely challenging computational problem. Values are obtained for the critical sliding or rotational speed and also for the exponential growth rate of each mode when operating above the critical speed.

In conventional clutch systems with alternating steel and composite discs, the dominant unstable mode is usually antisymmetric with respect to the steel discs, symmetric with respect to the composite discs, and involves an integer number of focal hot spots around the circumference. This prediction is confirmed by experimental observations of thermal damage in a five-disc clutch.

The method is easily applied to other examples and can therefore be used to assess the effect of design modifications such as changes in geometry and material properties on the thermoelastic stability of multi-disc brakes and clutches.

Y.-B.Y. and J.R.B. are pleased to acknowledge support from the National Science Foundation under contract no. CMS-9619527.

\section{Appendix A. Procedure for developing 'user elements'}

Circumferentially periodic displacement and temperature fields in an axisymmetric body can be discretized in the form

$$
\left.\begin{array}{rl}
u_{r} & =\sum_{i=1}^{N} W_{i} U_{r}^{i} \cos n \phi, \\
u_{\phi} & =\sum_{i=1}^{N} W_{i} U_{\phi}^{i} \sin n \phi, \\
u_{z} & =\sum_{i=1}^{N} W_{i} U_{z}^{i} \cos n \phi, \\
T & =\sum_{i=1}^{N} W_{i} \Theta_{i} \cos n \phi
\end{array}\right\}
$$

(Zienkiewicz 1989), where $W_{i}(r, z)$ is a set of $N$ shape functions defined in the two-dimensional cross-sectional domain, $\Omega$, and $U_{r}^{i}, U_{\phi}^{i}, U_{z}^{i}$ are the components of the corresponding nodal displacement vectors $\boldsymbol{U}_{i}$. The strain displacement relations in cylindrical polar coordinates can then be used to write the strain vector $\boldsymbol{e}=\left\{e_{r r}, e_{\phi \phi}, e_{z z}, e_{r \phi}, e_{\phi z}, e_{z r}\right\}^{\mathrm{T}}$ in the form

$$
\boldsymbol{e}=\sum_{i=1}^{N} \boldsymbol{B}_{i} \boldsymbol{U}^{i},
$$


where

$$
\boldsymbol{B}_{i}=\left[\begin{array}{ccc}
\frac{\partial W_{i}}{\partial r} \cos n \phi & 0 & 0 \\
\frac{W_{i}}{r} \cos n \phi & \frac{n W_{i}}{r} \cos n \phi & 0 \\
0 & 0 & \frac{\partial W_{i}}{\partial z} \cos n \phi \\
-\frac{n W_{i}}{2 r} \sin n \phi & \frac{1}{2}\left(\frac{\partial W_{i}}{\partial r}-\frac{W_{i}}{r}\right) \sin n \phi & 0 \\
0 & \frac{1}{2} \frac{\partial W_{i}}{\partial z} \sin n \phi & -\frac{n W_{i}}{2 r} \sin n \phi \\
\frac{1}{2} \frac{\partial W_{i}}{\partial z} \cos n \phi & 0 & \frac{1}{2} \frac{\partial W_{i}}{\partial r} \cos n \phi
\end{array}\right] .
$$

The corresponding stress vector $\boldsymbol{\sigma}=\left\{\sigma_{r r}, \sigma_{\phi \phi}, \sigma_{z z}, \sigma_{r \phi}, \sigma_{\phi z}, \sigma_{z r}\right\}^{\mathrm{T}}$ is given by the constitutive law

$$
\boldsymbol{\sigma}=\boldsymbol{C e}-\mathrm{DT}
$$

where

$$
C=\frac{E}{(1+\nu)(1-2 \nu)}\left[\begin{array}{cccccc}
1-\nu & \nu & \nu & 0 & 0 & 0 \\
\nu & 1-\nu & \nu & 0 & 0 & 0 \\
\nu & \nu & 1-\nu & 0 & 0 & 0 \\
0 & 0 & 0 & 1-2 \nu & 0 & 0 \\
0 & 0 & 0 & 0 & 1-2 \nu & 0 \\
0 & 0 & 0 & 0 & 0 & 1-2 \nu
\end{array}\right]
$$

and

$$
D=\frac{E \alpha}{(1-2 \nu)}\left\{\begin{array}{lllllll}
1 & 1 & 1 & 0 & 0 & 0
\end{array}\right\}^{\mathrm{T}} .
$$

Notice that the method can easily be extended to anisotropic materials by using appropriate anisotropic constitutive relations in place of equations (A 5) and (A 6). Substituting (A 2) into (A 4) and the resulting stresses into the weak (Galerkin) form of the equilibrium equations leads to the vector equation

$$
\boldsymbol{L} \boldsymbol{U}=\boldsymbol{G \Theta},
$$

where

$$
\begin{aligned}
\boldsymbol{L} & =\iiint_{\Omega} \boldsymbol{B}^{\mathrm{T}} \boldsymbol{C} \boldsymbol{B} r \mathrm{~d} r \mathrm{~d} z \mathrm{~d} \phi \\
\boldsymbol{G} & =\iiint_{\Omega} \boldsymbol{B}^{\mathrm{T}} \boldsymbol{D} \boldsymbol{W} \cos n \phi r \mathrm{~d} r \mathrm{~d} z \mathrm{~d} \phi,
\end{aligned}
$$

and $\boldsymbol{B}$ is the $6 \times 3 N$ matrix $\boldsymbol{B}=\left[\boldsymbol{B}_{1}, \ldots, \boldsymbol{B}_{N}\right]$. 
The integrals with respect to $\phi$ can be performed, using the result

$$
\int_{0}^{2 \pi} \cos ^{2} n \phi \mathrm{d} \phi=\int_{0}^{2 \pi} \sin ^{2} n \phi \mathrm{d} \phi= \begin{cases}\pi, & n \neq 0 \\ 2 \pi, & n=0\end{cases}
$$

giving the modified equation

$$
\tilde{\boldsymbol{L}} \boldsymbol{U}=\tilde{\boldsymbol{G}} \boldsymbol{\Theta}
$$

where

$$
\begin{aligned}
\tilde{\boldsymbol{L}} & =\iint_{\Omega} \tilde{\boldsymbol{B}}^{\mathrm{T}} \boldsymbol{C} \tilde{\boldsymbol{B}} r \mathrm{~d} r \mathrm{~d} z, \\
\tilde{\boldsymbol{G}} & =\iint_{\Omega} \tilde{\boldsymbol{B}}^{\mathrm{T}} \boldsymbol{D} \boldsymbol{W} r \mathrm{~d} r \mathrm{~d} z
\end{aligned}
$$

and $\tilde{\boldsymbol{B}}=\left[\tilde{\boldsymbol{B}}_{1}, \ldots, \tilde{\boldsymbol{B}}_{N}\right]$ with

$$
\tilde{\boldsymbol{B}}_{i}=\left[\begin{array}{ccc}
\frac{\partial W_{i}}{\partial r} & 0 & 0 \\
\frac{W_{i}}{r} & \frac{n W_{i}}{r} & 0 \\
0 & 0 & \frac{\partial W_{i}}{\partial z} \\
-\frac{n W_{i}}{2 r} & \frac{1}{2}\left(\frac{\partial W_{i}}{\partial r}-\frac{W_{i}}{r}\right) & 0 \\
0 & \frac{1}{2} \frac{\partial W_{i}}{\partial z} & -\frac{n W_{i}}{2 r} \\
\frac{1}{2} \frac{\partial W_{i}}{\partial z} & 0 & \frac{1}{2} \frac{\partial W_{i}}{\partial r}
\end{array}\right] .
$$

In these equations, $\tilde{\boldsymbol{L}}$ is the global stiffness matrix and $\tilde{\boldsymbol{G}} \boldsymbol{\Theta}$ is a vector of 'thermoelastic' nodal forces. The 'user element' option in a commercial finite-element code requires the user to evaluate and input the element stiffness matrix $\tilde{\boldsymbol{L}}_{\mathrm{e}}$, which has the same definition as (A 12) except that the integration is performed only over the element $\Omega_{\mathrm{e}}$, i.e.

$$
\tilde{\boldsymbol{L}}_{\mathrm{e}}=\iint_{\Omega_{\mathrm{e}}} \tilde{\boldsymbol{B}}^{\mathrm{T}} \boldsymbol{C} \tilde{\boldsymbol{B}} r \mathrm{~d} r \mathrm{~d} z .
$$

An essentially similar procedure can be used to define Fourier elements in the Cartesian coordinate system $x, y, z$.

\section{References}

Anderson, A. E. \& Knapp, R. A. 1990 Hot spotting in automotive friction systems. Wear 135, 319-337.

Banerjee, B. N. \& Burton, R. A. 1979 Experimental studies on thermoelastic effects in hydrodynamically lubricated face seals. ASME J. Lub. Technol. 101, 275-282. 
Barber, J. R. 1969 Thermoelastic instabilities in the sliding of conforming solids. Proc. R. Soc. Lond. A 312, 381-394.

Bryant, M. D., Wang, J. P. \& Lin, J. W. 1995 Thermal mounding in high speed dry slidersexperiment, theory and comparison. Wear 181, 668-677.

Burton, R. A., Nerlikar V. \& Kilaparti, S. R. 1973 Thermoelastic instability in a seal-like configuration. Wear 24, 177-188.

Christie, I., Griffiths, D. F., Mitchell, A. R. \& Zienkiewicz, O. C. 1976 Finite element methods for second order differential equations with significant first derivatives. Int. J. Numer. Meth. Engng 10, 1389-1396.

Dow, T. A. \& Stockwell, R. D. 1977 Experimental verification of thermoelastic instabilities in sliding contact. ASME J. Lub. Technol. 99, 359-364.

Du, S, Zagrodzki, P., Barber, J. R. \& Hulbert, G. M. 1997 Finite element analysis of frictionally excited thermoelastic instability. J. Thermal Stresses 20, 185-201.

Floquet, A. \& Dubourg, M. C. 1994 Non-axisymmetric effects for 3D thermal analysis of a brake. ASME J. Tribol. 116, 401-408.

Hartsock, D. L. \& Fash, J. W. 2000 Effect of pad/caliper stiffness, pad thickness and pad length on thermoelastic instability in disk brakes. ASME J. Tribol. (In the press.)

Inoue, H. 1986 Analysis of brake judder caused by thermal deformation of brake disk rotors. Society of Automotive Engineers, technical paper 865131.

Johansson, L. 1993 Model and numerical algorithm for sliding contact between two elastic halfplanes with frictional heat generation and wear. Wear 160, 77-93.

Kao, T. K., Richmond, J. W. \& Douarre, A. 2000 Brake disc hot spotting and thermal judder: an experimental and finite element study. Int. J. Vehicle Des. 23, 276-296.

Kennedy, F. E. \& Ling, F. F. 1974 A thermal, thermoelastic and wear simulation of a high energy sliding contact problem. ASME J. Lub. Technol. 96, 497-507.

Kreitlow, W., Schrödter, F. \& Matthäi, H. 1985 Vibration and hum of disc brakes under load. Society of Automotive Engineers, technical paper 850079.

Lee, K. \& Barber, J. R. 1993 Frictionally-excited thermoelastic instability in automotive disk brakes. ASME J. Tribol. 115, 607-614.

Lee, K. \& Barber, J. R. 1994 An experimental investigation of frictionally excited instability in automotive disk brakes under a drag brake application. ASME J. Tribol. 116, 409-414.

Lee, K. \& Dinwiddie, R. B. 1998 Conditions of frictional contact in disk brakes and their effects on brake judder. Society of Automotive Engineers, technical paper 980598.

Yeo, T. \& Barber, J. R. 1996 Finite element analysis of the stability of static thermoelastic contact. J. Thermal Stresses 19, 169-184.

Yi, Y.-B., Du, S., Barber, J. R. \& Fash, J. W. 1999 Effect of geometry on thermoelastic instability in disk brakes and clutches. ASME J. Tribol. 121, 661-666.

Zagrodzki, P. 1990 Analysis of thermomechanical phenomena in multidisc clutches and brakes. Wear 140, 291-308.

Zagrodzki, P., Lam, K. B., Al-Bahkali, E. \& Barber, J. R. 1999 Simulation of a sliding system with frictionally-excited thermoelastic instability. In Proc. 3rd Int. Cong. Thermal Stresses, Cracow, Poland, pp. 617-620.

Zienkiewicz, O. C. 1989 The finite element method, 4th edn. New York: McGraw-Hill. 